Écrire

l'histoire

\section{Écrire l'histoire}

Histoire, Littérature, Esthétique

$3 \mid 2009$

Le détail (1)

\title{
Mémoire involontaire et détail mnémotechnique
}

Czapski lecteur de Proust, camp de Griazowietz, URSS, 1941

\section{Guillaume Perrier et Agnieszka Zuk}

\section{OpenEdition}

Journals

Édition électronique

URL : http://journals.openedition.org/elh/937

DOI : $10.4000 /$ elh.937

ISSN : 2492-7457

Éditeur

CNRS Éditions

\section{Édition imprimée}

Date de publication : 1 juin 2009

Pagination : 44-54

ISBN : 978-2-35698-008-3

ISSN : 1967-7499

Référence électronique

Guillaume Perrier et Agnieszka Zuk, « Mémoire involontaire et détail mnémotechnique », Écrire l'histoire [En ligne], 3 | 2009, mis en ligne le 01 juin 2012, consulté le 23 septembre 2020. URL : http:// journals.openedition.org/elh/937 ; DOI : https://doi.org/10.4000/elh.937 


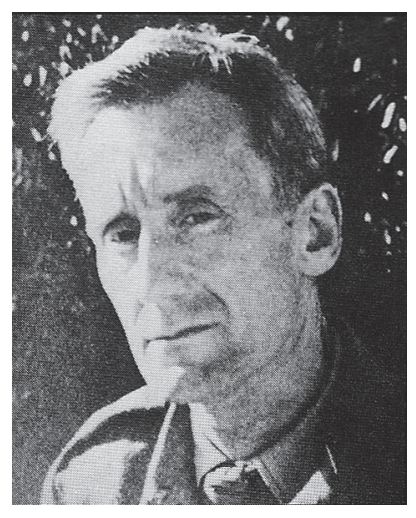

Joseph Czapski en 1943 


\section{Mémoire involontaire}

\section{et détail mnémotechnique}

\section{Czapski lecteur de Proust, camp de Griazowietz, URSS, 1941}

P $_{\text {Ierre Vidal-Naquet assignait naguère à }}$ l'historien la tâche de «mettre en mouvement la mémoire, [de] faire en somme pour l'histoire ce que Proust avait fait pour le roman ${ }^{1} »$. Il se référait à Shoah de Claude Lanzmann, mais on pourrait faire remonter l'origine de cette méthode à l'expérience de Joseph Czapski (18961993), peintre et intellectuel polonais, lecteur assidu de Proust pendant l'entre-deux-guerres, détenu dans plusieurs camps de prisonniers soviétiques entre 1939 et 1941. Pour entretenir une forme d'activité intellectuelle et résister à l'anéantissement moral, lui et ses camarades officiers de l'armée polonaise organisèrent une série de conférences sur les sujets les plus divers: peinture, architecture, bibliophilie ou encore alpinisme - cela, sans disposer d'aucun livre ${ }^{2}$.

\begin{abstract}
« Chacun de nous parlait de ce dont il se souvenait le mieux », écrit Czapski, qui, pour sa part, choisit entre autres thèmes l'œuvre de Marcel Proust ${ }^{3}$.

Le texte des conférences sur l'auteur d'À la recherche du temps perdu, prononcées début 1941, est paru d'abord en 1948 en traduction polonaise puis en 1987 dans la version originale française, illustré de certaines pages extraites du journal manuscrit de Czapski et reproduites en facsimilé $^{4}$. Certaines de ces pages, contemporaines des conférences, permettent de mieux comprendre la performance orale et mnémonique réalisée par le prisonnier polonais. Elles sont remplies de notes non rédigées, allant de deux ou trois mots jusqu'à six, sept lignes, écrites en langue polonaise à l'exception des noms propres français et de quelques expressions ponctuelles. Certaines
\end{abstract}

1. Pierre Vidal-Naquet, Les fuifs, la mémoire et le présent, Paris, La Découverte, 1991, vol. II, p. 221.

2. Pierre Pachet, "Quand il n’y a plus de livres », La Quinzaine littéraire, no 905, 1-31 août 2005, p. 29.

3. Joseph Czapski, Proust contre la déchéance. Conférences au camp de Griazowietz, Lausanne, Éditions Noir sur Blanc, 1987.

4. Joseph Czapski, «Proustw Griazowcu», Kultura, no 12-13, 1948, repris dans Joseph Czapski, Czytajac, Cracovie, Znack, 1990, p. 108-163; id., Proust contre la déchéance..., op. cit. 
notes sont numérotées, suivant une démarche qui semble chercher à ordonner les souvenirs notés au fur et à mesure qu'ils sont venus à l'esprit. Chaque page ne correspond pas à une partie du texte des conférences, mais reprend presque toute leur matière et l'agence de différentes manières. Certains mots sont soulignés d'un trait coloré, entourés, voire reliés les uns aux autres par d'autres traits. Sur une double page, en particulier, on peut voir une représentation graphique des différents thèmes et composantes du roman de Proust, où Czapski met en œuvre, sinon ses qualités de peintre, du moins sa prédisposition à visualiser ses idées ${ }^{5}$. De part et d'autre d'un gros point noir où se rejoignent tous les traits apparaissent deux mots en français, «cloison étanche », détail en apparence insignifiant mais qui attire l'attention par sa position centrale dans le schéma: juste sous le titre Du côté de chez Swann - premier volume du roman de Proust où sont amorcés tous les thèmes - et sous l'expression « composition gothique » en polonais, qui désigne le modèle architectural de son livre selon Proust lui-même. À travers l'interprétation de ce « détaildettaglio ${ }^{6} »$, notre intention est de comprendre comment Czapski a pu se remémorer l'immense roman de Proust sans le secours d'aucun livre, mais aussi d'envisager l'usage qu'il a fait de la mémoire proustienne dans le contexte du camp soviétique de Griazowietz.

La formule «cloison étanche» ne figure pas dans le texte des conférences: aucune explication ne permet de comprendre pourquoi le conférencier lui accorde une telle importance dans ses notes. La première hypothèse serait que Cizapski emprunte ces deux mots au texte de Proust. En effet, «cloison» est un mot proustien, employé précisément dans le contexte des expériences de mémoire involontaire. Il joue un rôle particulièrement important dans l'épisode de réminiscence malheureuse des «Intermittences du cœur », où le héros prend conscience que sa grand-mère est morte. Proust emploie ce mot à plusieurs reprises dans le même passage, pour désigner le mur qui séparait - et réunissait - la chambre de la grand-mère et celle du héros au Grand Hôtel de Balbec. Autrefois ils communiquaient en frappant de petits coups complices contre ce mur, mais désormais:

Je n'osais pas approcher de cette cloison plus que d'un piano où ma grand'mère aurait joué et qui vibrerait encore de son toucher. ${ }^{7}$

Le syntagme entier «cloison étanche » apparaît deux fois dans $\grave{A}$ la recherche du temps

5. Id., Proust contre la déchéance..., op. cit., p. 62-63.

6. Détail qui porte la «trace de l'action de celui qui fait le détail » : Daniel Arasse, Le Détail. Pour une histoire rapprochée de la peinture, Paris, Flammarion (Idées et recherches), 1992, p. 11.

7. Marcel Proust, Sodome et Gomorrhe, dans À la recherche du temps perdu, éd. par J.-Y. Tadié, Paris, Gallimard (Bibliothèque de la Pléiade), 1987-1989, vol. III, p. 159 (nous soulignons). 


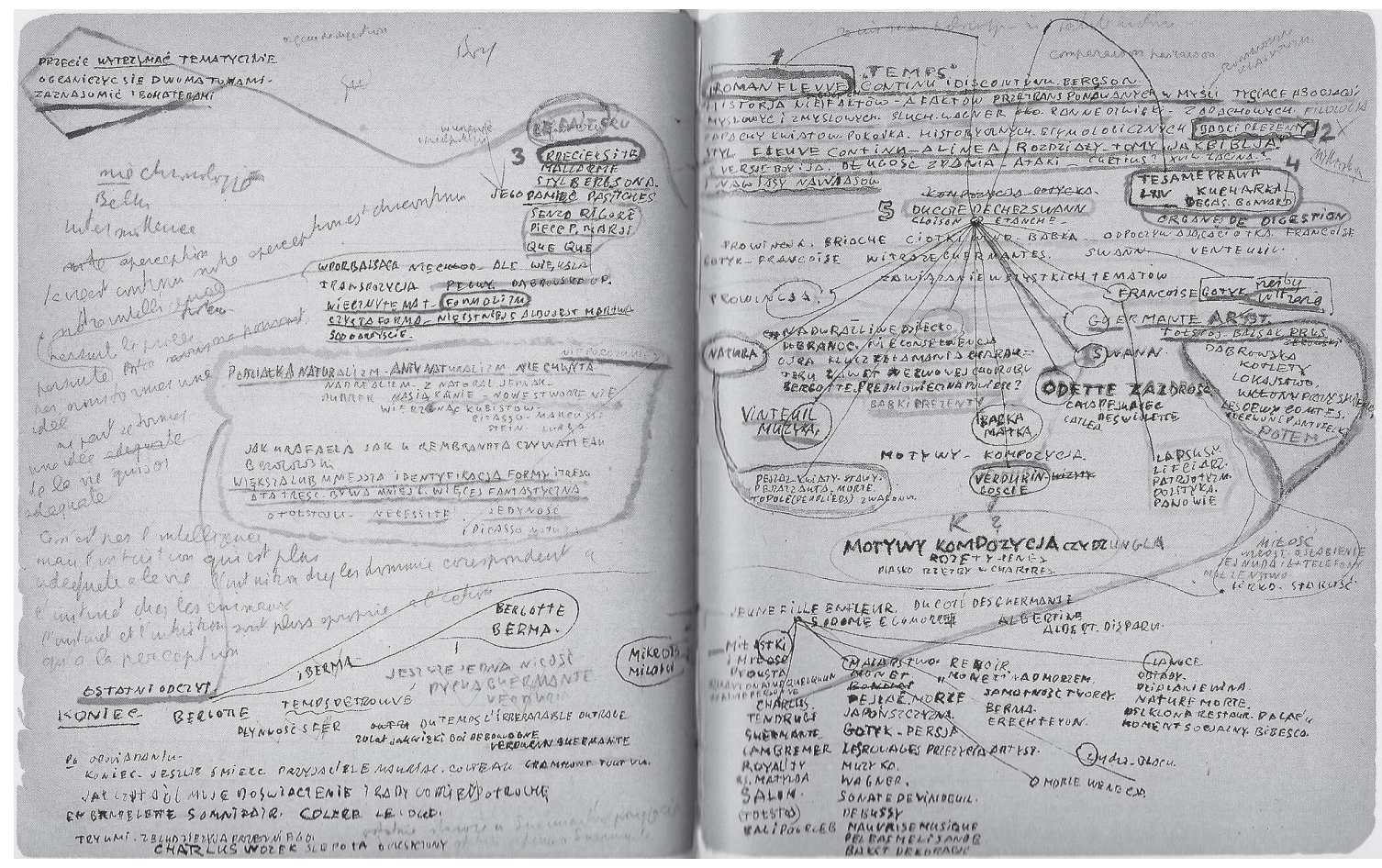

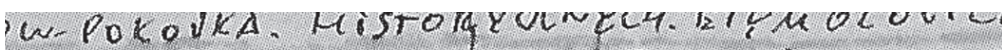
CoNtiNH-A L-i

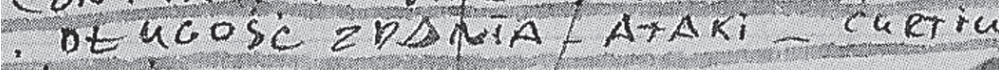
$\operatorname{vis} \Delta$ b

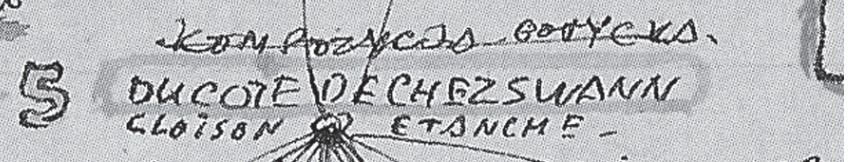

GRiOCHE CIOTKTIWWO-BSAKA ODPO

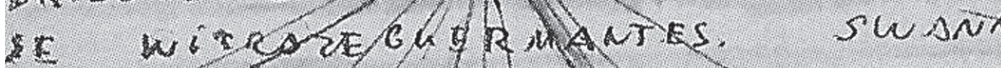

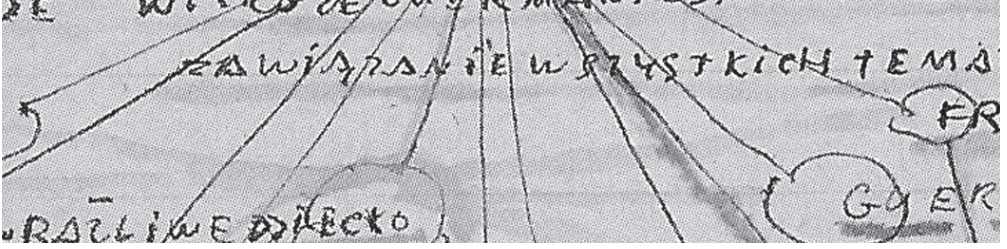

Extrait du journal de Czapski (avec l'aimable autorisation des Éditions Noir sur blanc)

Guillaume Perrier et Agnieszka Zuk, «Czapski lecteur de Proust, camp de Griazowietz, URSS, 1941 » Écrire l'histoire, $n^{\circ} 3$ - printemps 2009, 44-54 
perdu: la première fois dans Le Côté de Guermantes, à propos de la double vie de l'oncle Adolphe, qui se brouille avec le reste de sa famille parce qu'il fréquente des cocottes et des actrices, la seconde fois dans Le Temps retrouvé, à propos des recherches angoissées du héros pour connaître les penchants sexuels de sa maîtresse défunte, Albertine. Dans un mouvement de la pensée qui vise à se rassurer et à innocenter Albertine de relations homosexuelles, le héros-narrateur se dit:

Elle avait peut-être vécu près de l'amie de $\mathrm{M}^{\mathrm{lle}}$ Vinteuil et d'Andrée, séparée par une cloison étanche d'elles qui croyaient qu'elle «n'en était pas ». ${ }^{8}$

Avec ces réflexions tortueuses sur la vie secrète des individus, que ce soit Albertine ou l'oncle du héros, nous sommes très loin en apparence de Czapski et des camps soviétiques. Mais si on lit la phrase qui précède celle que nous venons de citer, un mot saute aux yeux: Albertine se serait prévalue d'avoir fréquenté ces femmes homosexuelles « pour ne pas avoir l'air de ne pas savoir ce que c'était, comme dans une conversation on prend un air entendu si on parle de Fourier ou de Tobolsk, encore qu'on ne sache pas ce que c'est ${ }^{9}$ ». En lisant cette phrase, Czapski a dû s'amuser du trait d'esprit de Proust, sachant bien que c'était à Tobolsk, en Sibérie, que le tsar Nicolas II et sa famille avaient été internés de l'automne 1917 au printemps 1918, avant d'être assassinés. Czapski suivait l'actualité révolutionnaire de près et se trouvait précisément en Russie en 1917, dans un régiment de soldats polonais puis dans une communauté religieuse à Petrograd. Le sentiment de complicité et l'amusement suscités par l'allusion de Proust ont pu être un facteur de mémorisation de ce passage et, par contiguité, de l'expression « cloison étanche ».

Cela peut expliquer que Czapski ait mémorisé cette expression mais ne nous dit pas pourquoi il a fait de ce détail une « formule » qui se trouve au centre de son schéma mnémotechnique de l'œuvre proustienne ${ }^{10}$. Pour l'élucider, à défaut de trouver une réponse dans le texte des conférences, il faut chercher dans d'autres écrits de Czapski. Or, la même expression apparaît, associée également au nom de Proust, à peine quatre ans plus tard, dans le texte programmatique de la collection «Témoignages » fondée par Czapski en 1945, peu avant la fin de la guerre. Ce texte est imprimé sur un feuillet rose, collé dans l'édition originale du premier livre de la collection, Souvenirs de Starobielsk de Czapski lui-même :

Il n'y a plus de spectateurs dans les pays frappés par la guerre. Tout le monde y participe, non seulement le soldat de l'armée mais celui du maquis, femmes, enfants,

8. Marcel Proust, Le Temps retrouvé, dans À la recherche du temps perdu, op. cit., vol. IV, p. 286 (nous soulignons); id., Le Côté de Guermantes, ibid., vol. II, p. 561.

9. Id., Le Temps retrouvé, op. cit., p. 286 (nous soulignons).

10. Nous empruntons la notion de « formule » à Marcel Jousse, Le Style oral. Rythmique et mnémotechnique chez les verbo-moteurs [1924], Paris, Fondation Marcel Jousse, 1981 ; id., L'Anthropologie du geste [1969], Paris, Gallimard (Tel), 2008. 
des villes bombardées, vieillards déportés de même que tous les écrivains. Les cloisons étanches qui protégeaient la croissance de l'œuvre de Proust pendant la dernière guerre ont été rasées. [...] Il est encore difficile d'affirmer que nous soyons les témoins de l'éclosion d'une nouvelle attitude morale de l'écrivain envers les faits, mais il n'y a aucun doute que le développement tel qu'il est déterminé par la guerre actuelle implique plus d'importance aux faits qu'à la fiction littéraire car l'auteur se souvient des milliers d'êtres voués au silence éternel et d'autres incapables d'exprimer ce qu'ils ont vécu. [...] Le but de cette collection est de nous faire revivre un monde vécu par les hommes ressenti et non déformé par les écrivains, pour que nous nous en souvenions en créant des faits nouveaux. ${ }^{11}$

Ici, la formule «cloisons étanches » met l'œuvre de Proust à distance, la comparant implicitement à une plante de serre et faisant allusion à la croissance d'À la recherche du temps perdu durant la Première Guerre mondiale, à l'abri des combats: la guerre a retardé la publication des volumes postérieurs à $D u$ côté de chez Swann (1913) et a permis à Proust d'amplifier considérablement son œuvre par rapport à son projet initial, qui devait tenir en deux ou trois volumes. Czapski, qui a lu des études critiques sur la Recherche et une partie de la correspondance de son auteur, est bien renseigné sur ces données génétiques de la création proustienne ${ }^{12}$. Il devait s'en souvenir à l'époque des conférences, d'autant plus amèrement qu'il craignait pour sa propre œuvre de peintre, qui représentait vingt années de labeur et qui a été effectivement anéantie durant la Seconde Guerre mondiale. On peut citer à ce sujet Murielle Werner-Gagnebin, spécialiste de l'œuvre picturale de Czapski :

À Griazowietz, il réussit à se procurer quelques godets d'aquarelle et il tente d'esquisser en raccourci ses dernières toiles, exécutées avant la guerre. C'est sur ces seuls témoins, ainsi que sur quelques rares photographies échappées au massacre, que nous pouvons, aujourd'hui, nous fonder pour entreprendre l'étude de ce que fut la vision « initiale » d'un peintre qui à quarante-trois ans perdit, d'un seul coup, la totalité de son æuvre. ${ }^{13}$

Le travail de témoignage que Czapski assigne à l'écrivain à la fin de la guerre se démarque donc de Proust. L'artiste est alors exposé directement aux souffrances des combats ou de l'internement: avenir incertain, mauvais traitements, sousalimentation, manque de vêtements dans les grands froids du Nord. Cela n’a pas empêché Czapski de méditer l'œuvre de Proust en 1941 et de publier ses conférences en 1948, longtemps avant la réhabilitation de l'auteur de la Recherche dans les milieux intellectuels français. Dans le texte fondateur des Temps modernes, au sortir de la guerre, Sartre déclarait: «Nous ne croyons

11. Joseph Czapski, Souvenirs de Starobielsk, Paris, Collection Témoignages, 1945; le feuillet figure dans le volume conservé par la BNF sous la cote 16-Z-27834 (1). Réédition, sans le texte programmatique, aux Éditions Noir sur Blanc, Lausanne, 1987 (nous soulignons).

12. Id., Proust contre la déchéance..., op. cit., p. 53.

13. Murielle Werner-Gagnebin, Czapski: la main et l'espace, Lausanne, L’Âge d'homme, 1974, p. 153. 
plus à la psychologie intellectualiste de Proust, et nous la tenons pour néfaste ${ }^{14}$. » Au contraire, Czapski ne manifeste aucun ressentiment dans ses conférences, même quand il mentionne le confort de Proust, qui devait paraître extravagant par rapport aux conditions de vie du camp. Il évoque à ses camarades transis de froid la «chambre surchauffée » de l'écrivain malade, ses chemises couvertes de «taches brunes de brûlure parce qu'il les chauffe "à brun" avant de les mettre ${ }^{15}$ ». L'idée de «cloison étanche» entendue comme séparation protectrice transparaît également à travers la mention du « liège sur les murs », destiné à insonoriser la chambre de Proust, ou encore dans le récit de sa retraite pour accomplir son œuvre:

La claustration de ce dernier est alors déjà de plus en plus absolue, à cause de ses malheurs personnels, son labeur énorme, sa maladie. Possédé par son œuvre, il la poursuit avec une indépendance absolue des courants artistiques d'alors. ${ }^{16}$

Sa vie évolue dans la solitude, derrière une «cloison étanche» qui sépare l'écrivain du monde.

Les prisonniers de Griazowietz connaissent une autre forme de claustration, mais dans ses conférences Czapski insiste toujours sur la maladie et le labeur de Proust, sans jamais les relativiser ni les minimiser. Il évoque par ailleurs le fait que, se sachant condamné, l'auteur de la Recherche sacrifie sciemment les dernières années de sa vie à son œuvre:

Proust ne vit plus que pour son œuvre, et sa vie n'a désormais d'autre fonction que celle d'alimenter l'œuvre naissante [...]. Proust n'aspire plus à vivre en dehors de l'œuvre, il a vécu sa vie. Est-il heureux? Mais il ne se pose certainement pas cette question. Une seule chose lui importe: terminer à temps, vaincre la course contre la mort. ${ }^{17}$

\section{Ou encore :}

L'auteur a soudain la révélation du sens de son existence: toutes ses souffrances et ses joies avaient un seul et unique sens, celui de lui donner la pleine conscience de la vie et des lois qui la régissent.

La notion de «cloison étanche » met-elle en rapport l'enfermement, l'isolement, de Proust et ceux de Czapski et ses camarades? Coupé du monde et de la vie par sa maladie, Proust continue à créer en puisant dans sa mémoire et ne sort que très rarement pour vérifier quelque détail manquant. C'est aussi grâce à la mémoire que, contraints à survivre dans des conditions inhumaines, les détenus peuvent se tourner vers un monde dont ils ont été exclus et qu'ils craignent de ne jamais revoir.

En essayant d'établir un parallèle entre la tragédie de Proust, d'une part, la sienne et celle

14. Jean-Paul Sartre, «Présentation des Temps modernes », Les Temps modernes, n 1, octobre 1945; id., Situations II. Littérature et engagement [1948], Paris, Gallimard, 1999, p. 20.

15 Joseph Czapski, Proust contre la déchéance..., op. cit., p. 9, 33.

16 Ibid., p. 21, 64, 94.

17. Joseph Czapski, Czytajac, op. cit., p. 171 (trad. A. Zuk). 
de ses codétenus, d'autre part, Czapski auraitil cherché à donner un sens à leur souffrance, à les inciter à résister intérieurement, le plus difficile étant pour un détenu de préserver sa part d'humanité? Remarquons que ni Czapski ni aucun de ses camarades ne choisissent pour sujet de leurs conférences les grands écrivains polonais du siècle précédent, tels Adam Mickiewicz, Juliusz Slowacki ou Henryk Sienkiewicz. Le choix de ces auteurs, qui ont souvent inspiré les Polonais lors des périodes d'occupation, de guerre ou d'exil, n'aurait surpris personne. Mais les sujets des conférences de Griazowietz visent d'abord l'humain, l'individuel, et non le patriote ou le Polonais en chacun des détenus. Un dialogue s'établit ainsi à travers le temps entre le prisonnier et l'écrivain qui a perdu au front certains de ses amis parmi les plus chers et qui écrivait en 1914:

Je pleure la mort de tout le monde, même des gens que je n'ai jamais vus. C'est un sens que nous a ajouté la guerre, par l'exercice effroyable de l'angoisse quotidienne, celui qui fait souffrir pour des inconnus. ${ }^{18}$

Czapski affirme en outre, dans la préface de la traduction polonaise (1948), que c'est à Griazowietz qu'il a compris le sens profond de l'œuvre proustienne:
Loin de tout ce que pouvait me rappeler le monde de Proust, mes souvenirs de lui, qui au départ me semblaient extrêmement minces, ont commencé soudain et de façon inattendue à croître avec une force et une précision qui semblaient indépendantes de ma volonté. ${ }^{19}$

L'idée de croissance est associée au nom de Proust, comme dans l'argument de la collection «Témoignages », mais elle désigne cette fois l'expérience mnémonique de Czapski lui-même. La suite de la préface confirme cette analogie, dans la mesure où il reprend l'image proustienne des petits papiers japonais se déployant dans l'eau, qui caractérisait le souvenir déclenché par la madeleine dans $D u$ côté de chez Swann ${ }^{20}$. La «cloison étanche » du camp a donc permis à Czapski de faire lui-même l'expérience de la mémoire involontaire.

On comprend mieux ainsi pourquoi cette formule se trouve au centre de la représentation graphique du manuscrit - alors que la célèbre madeleine, altérée par la mémoire sous le nom de «brioche », occupe une place insignifiante dans le même schéma, au début d'une énumération des réminiscences proustiennes: «Brioche, dalles, serviette, la mort indifférente, hymne à la joie ${ }^{21}$ ». Les deux mots magiques de "cloison étanche ", tel un sésame, permettent de condenser toute l'ambivalence, aussi bien la tension que la

18. Marcel Proust, Correspondance, éd. Ph. Kolb, Paris, Plon, 1971-1993, vol. XVI, p. 272.

19. Joseph Czapski, Czytajac, op. cit., p. 108 (trad. A. Zuk).

20. Marcel Proust, Du côté de chez Swann, dans À la recherche du temps perdu, op. cit., vol. I, p. 47.

21. Joseph Czapski, Proust contre la déchéance..., op. cit., p. 62. 
sympathie profonde, qui anime le rapport de Czapski à Proust. Ils articulent ses souvenirs de lecture et activent sa mémoire orale et vivante. Ce n'est pas une expression purement référentielle, qui désignerait un motif minuscule de la Recherche, mais un embrayeur qui inscrit l'œuvre de Proust dans le contexte du camp de prisonniers et de la guerre. C'est le déclencheur de la «mémoire involontaire » de Czapski.

Non seulement un détail contingent permet de déployer le souvenir de l'œuvre de Proust, mais a posteriori le texte des conférences permet de faire vivre les circonstances dans lesquelles elles ont été prononcées. En 1944, projetant de publier ses conférences, Czapski écrit en introduction:

Je vois encore mes camarades entassés sous les portraits de Marx, Engels et Lénine, harassés après un travail dans un froid qui montait jusqu'à quarante-cinq degrés, qui écoutaient nos conférences sur des thèmes tellement éloignés de notre réalité d'alors. ${ }^{22}$

Il ajoute :

Sur ce fond lugubre, ces heures passées avec des souvenirs sur Proust, Delacroix, me semblent les heures les plus heureuses.

Le contraste est au principe même de l'impression mémorable, comme dans $D u$ côté de chez Swann, au début du passage sur la lecture à Combray, où l'obscurité de la chambre contient elle-même la lumière éclatante, extérieure, de l'été ${ }^{23}$. Dans la théorie proustienne, toute la valeur d'un livre réside non dans son contenu mais dans les circonstances de sa lecture, que le livre permet de percevoir et surtout de se remémorer plus intensément:

Il n'y a peut-être pas de jours de notre enfance que nous ayons si pleinement vécus que ceux que nous avons cru laisser sans les vivre, ceux que nous avons passés avec un livre préféré. ${ }^{24}$

Czapski, lecteur sans livre, illustre cette théorie paradoxale de la lecture, à ceci près que son expérience ne se produit pas dans le milieu protégé de la famille et de l'enfance, mais dans l'univers chaotique et violent de la guerre. La remémoration dépasse le cadre immédiat des conférences pour s'étendre au souvenir des camarades dont la disparition préoccupait Czapski et ses codétenus à la même époque:

Il nous est incompréhensible pourquoi justement nous, quatre cents officiers et soldats, fûmes sauvés sur quinze mille camarades qui disparurent sans traces. ${ }^{25}$

Ces disparus n'étaient autres que les victimes des massacres de Katyn, dont le sort était encore incertain en 1941, à l'époque des conférences,

22. Ibid., p. 9 .

23. Marcel Proust, Du côté de chez Swann, op. cit., p. 82.

24. Marcel Proust, «Journées de lecture », Pastiches et mélanges, dans Contre Sainte-Beuve, éd. P. Clarac et Y. Sandre, Paris, Gallimard (Bibliothèque de la Pléiade), 1985, p. 160.

25. Joseph Czapski, Proust contre la déchéance..., op. cit., p. 9. 


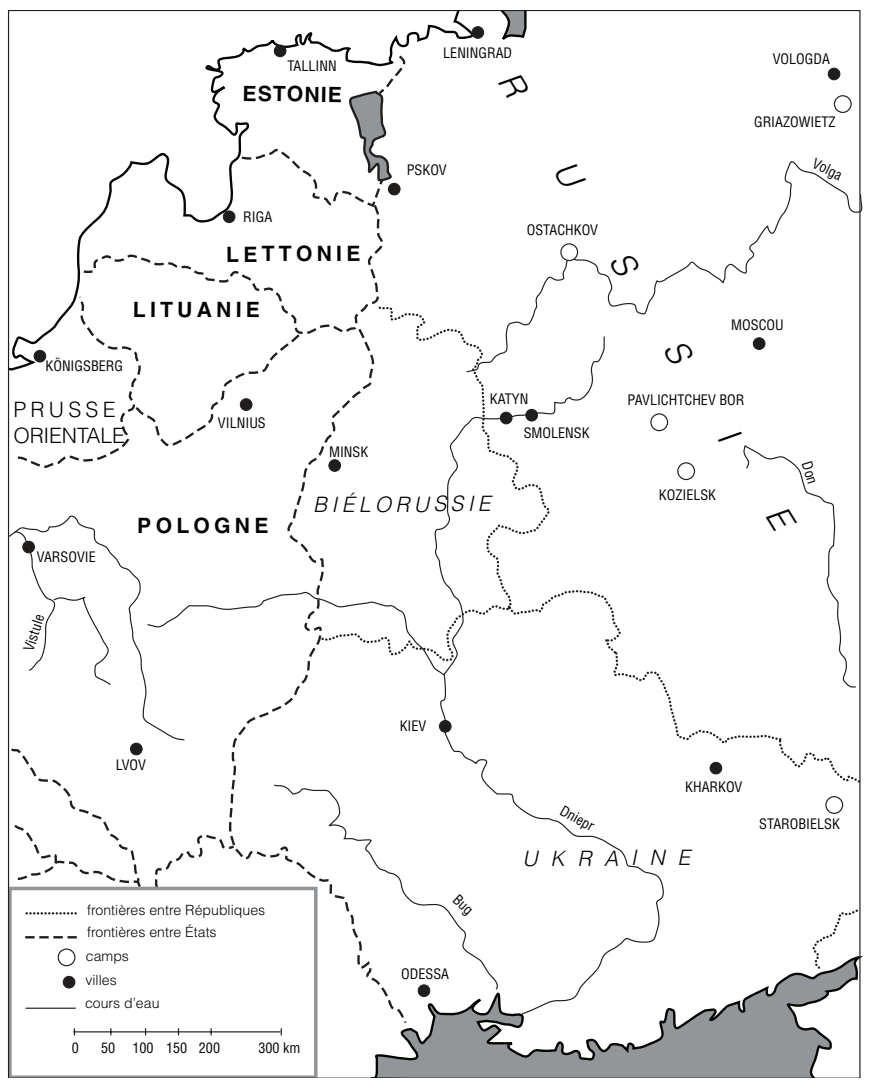

L'Europe centrale dans ses frontières de 1937

les charniers n'ayant été découverts que progressivement, d'août 1941 à 1944.

Les prisonniers de Griazowietz étaient, dans une certaine mesure, les survivants de Katyn - événement qui marqua leur vie de manière indélébile et celle de Czapski plus que toute autre. Après l'invasion de la Pologne menée conjointement par l'Union soviétique et
l'Allemagne nazie en septembre 1939, Czapski fut interné successivement dans trois camps d'URSS : Starobielsk, dans l'est de l'Ukraine, Pavlichtchev, au sud-ouest de Moscou, et Griazowietz, encore plus au nord, près de la ville de Vologda. Sur les quatre mille prisonniers de Starobielsk, il y eut soixante-dix-neuf survivants, ceux-là mêmes qui se retrouvèrent dans le camp de Griazowietz. 
Les autres, regroupés avec des prisonniers venus de Kozielsk et d'Ostachkov, furent massacrés dans la tristement célèbre forêt de Katyn. Dans l'armée polonaise reconstituée en août 1941 par Staline, après l'attaque de Hitler contre l'URSS et le retournement des alliances, Czapski occupa le poste de «chef de la Propagande », c'est-àdire responsable des publications et des activités culturelles, mais il fut également envoyé en mission diplomatique en Union soviétique pour retrouver la trace de ses anciens camarades disparus. Les nombreuses démarches qu'il entreprit auprès des autorités moscovites restèrent lettre morte et renforcèrent dans le même temps sa défiance envers l'opinion qui prévalait alors chez les Alliés, à savoir la culpabilité des nazis. Ni Roosevelt ni Churchill, soucieux de s'attirer les bonnes grâces de Staline, ne permettaient alors que fût accréditée l'implication des Soviétiques.
Dans ce contexte, la théorie proustienne de la mémoire et de la lecture apparaît comme un moyen pour Czapski de maintenir vivant le souvenir des camps et des disparus, menacés d'oubli par la grande Histoire. Elle a pu contribuer à ce qu'il développe une contre-histoire ou une micro-histoire, basée sur le détail et la recherche des indices ${ }^{26}$, et à ce qu'il fasse œuvre d'écrivain à son tour dans Souvenirs de Starobielsk puis, surtout, dans Terre inhumaine, livres dans lesquels il raconte sa quête désespérée des victimes de Katyn et défend la thèse de la culpabilité des Soviétiques. L'un des rares soutiens de Czapski en France était l'historien Daniel Halévy. Cet ami intime et camarade de classe de Proust au lycée Condorcet à la fin des années 1880, premier biographe de Nietzsche en France, avait rencontré Czapski à Paris pendant l'entre-deux-guerres et préfaça Terre inhumaine en $1948{ }^{27}$.

Nous remercions $M^{\text {me }}$ Carine Trevisan pour son rôle déterminant dans l'élaboration de cet article.

26. Carlo Ginzburg, « Traces. Racines d'un paradigme indiciaire », dans Mythes, emblèmes, traces: morphologie et histoire, trad. M. Aymard, Chr. Paolini, E. Bonan, M. Sancini-Vignet, Paris, Flammarion, 1989, p. 178: «On peut aisément démontrer que le plus grand roman de notre temps $-\grave{A}$ la recherche du temps perdu - est construit selon un rigoureux paradigme indiciaire. » 27. Joseph Czapski, Terre inhumaine [1949], trad. A. M. Bohomolec, Lausanne, L’Âge d'homme (Les Chemins effacés), 1991. 